\title{
Euclidean Geodesic Loops on High-Genus Surfaces Applied to the Morphometry of Vestibular Systems
}

\author{
Shi-Qing Xin ${ }^{1}$, Ying $\mathrm{He}^{1}$, Chi-Wing Fu${ }^{1}$, Defeng Wang ${ }^{2}$, Shi $\mathrm{Lin}^{2}$, \\ Winnie C.W. Chu ${ }^{2}$, Jack C.Y. Cheng ${ }^{3}$, Xianfeng $\mathrm{Gu}^{4}$, and Lok Ming Lui ${ }^{5}$ \\ 1 School of Computer Engineering, Nanyang Technological University \\ 2 Department of Imaging and Interventional Radiology, CUHK \\ 3 Department of Orthopaedics and Traumatology, CUHK \\ 4 Department of Computer Science, Stony Brook University \\ 5 Department of Mathematics, The Chinese University of Hong Kong
}

\begin{abstract}
This paper proposes a novel algorithm to extract feature landmarks on the vestibular system (VS), for the analysis of Adolescent Idiopathic Scoliosis (AIS) disease. AIS is a 3-D spinal deformity commonly occurred in adolescent girls with unclear etiology. One popular hypothesis was suggested to be the structural changes in the VS that induce the disturbed balance perception, and further cause the spinal deformity. The morphometry of VS to study the geometric differences between the healthy and AIS groups is of utmost importance. However, the VS is a genus-3 structure situated in the inner ear. The high-genus topology of the surface poses great challenge for shape analysis. In this work, we present a new method to compute exact geodesic loops on the VS. The resultant geodesic loops are in Euclidean metric, thus characterizing the intrinsic geometric properties of the VS based on the real background geometry. This leads to more accurate results than existing methods, such as the hyperbolic Ricci flow method [13. Furthermore, our method is fully automatic and highly efficient, e.g., one order of magnitude faster than [13. We applied our algorithm to the VS of normal and AIS subjects. The promising experimental results demonstrate the efficacy of our method and reveal more statistically significant shape difference in the VS between right-thoracic AIS and normal subjects.
\end{abstract}

\section{Introduction}

In medical image analysis, surface-based morphometry has been commonly applied for disease analysis. For example, in human brain mapping, neuroscientists are interested in detecting shape changes or abnormalities on brain cortical surfaces for analyzing various brain diseases. Hippocampal surface morphometry has also been an active research area for the analysis of Alzheimer's disease.

Although surface-based shape analysis has been extensively studied, most existing methods can only deal with surfaces with simple topology. For example, both the brain cortical surface and the hippocampal surface are of genus 0 . Dealing with high-genus surfaces are apparently much more difficult, due 
to their complicated topology. One typical example of high-genus anatomical structures is called the vestibular system (VS). The VS is a genus-3 structure situated in the inner ear, which is responsible for detecting head movements and sending postural signals to the brain. The morphometry of VS plays an important role in the analysis of various diseases such as the Adolescent Idiopathic Scoliosis (AIS) disease. AIS is a $3 D$ spinal deformity which affects about $4 \%$ schoolchildren worldwide. The etiology of AIS is still unclear but believed to be a multi-factorial disease. One popular hypothesis was suggested to be the structural changes in the VS that induce the disturbed balance perception, and further cause the spinal deformity 149$]$. Some recent works have also revealed the statistical difference in global morphology of the VS between right-thoracic AIS and normal controls 813. Hence, analyzing the shape of VS is crucial for understanding AIS. However, since the VS has high-genus topology, it poses great challenge for shape analysis, and an effective algorithm is thus needed.

In this work, we proposed a novel algorithm to effectively extract feature landmarks on the VS, for the disease analysis of AIS. The basic idea is to compute exact geodesic loops on the VS. The resultant geodesic loops are in Euclidean metric, thus characterizing the intrinsic geometric properties of the VS based on the real background geometry. This leads to more accurate results than the existing methods, such as the hyperbolic Ricci flow method [13]. Furthermore, our method is fully automatic and highly efficient, e.g., one order of magnitude faster than 13. To test the effectiveness of our proposed method, we applied the algorithm to the VS of normal and AIS subjects. Experimental results demonstrate the efficacy of our method and reveal more statistically significant shape differences in the VS between right-thoracic AIS and normal subjects.

\section{Related Work}

Shi et al. 8] proposed to consider the radii of the canals for the global morphology of the VS. The drawback was that the complete geometry of the surface has not been fully analyzed. In [13, Zeng et al. proposed to extract the geodesic spectra of the VS to compare geometric difference between the normal and unhealthy groups. This algorithm requires the computation of uniformization metric, which is computed by discrete Ricci flow or Yamabe flow [5]. Since both curvature flows are highly non-linear PDEs, the computational costs are very high. It is known that embedding surfaces of genus $g \geq 2$ onto the hyperbolic space $\mathbb{H}^{2}$ could be error prone due to the numerical truncation, the input meshes must come with good quality (i.e., the vertices are distributed uniformly over the surface) and contain small number of vertices (usually a few thousands). Compared to this approach, our method is computationally efficient and numerically stable. Furthermore, the computed geodesic of [13] was based on the hyperbolic metric and hence it was not reflecting the real background geometry of the VS.

In this paper, we suggest computing $2 g$ characteristic geodesic loops and use their lengths for the purpose of AIS disease analysis. A discrete geodesic on a polyhedral surface in $\mathbb{R}^{3}$ is defined to be a polyline that is locally shortest 
everywhere [7. In detail, Mitchell et al. 6] pointed out the requirements of a discrete geodesic $l$ : 1) For each face $f$, the intersection between $f$ and $l$ must be empty or a line segment; 2) For each edge that $l$ intersects, the entry angle must equal to the departure angle; and 3) For each vertex that $l$ passes through, the two angles passed through by $l$ must be no less than $\pi$.

Closed geodesics are also called geodesic loops. Though defined on polygonal meshes, discrete geodesics may pass through the face interior, rather than restricted on mesh edges. They are intrinsic to geometry and insensitive to the mesh tessellation and resolution. Therefore, the resultant spectra depend on the actual geometry of the input VS geometry, independent of the triangulation.

To our knowledge, very few algorithms are known for the geodesic loop problem. Wu and Tai [1] proposed the discretized geodesic curvature flow (dGCF) to compute geodesic loops on triangle meshes using a level set formulation. Later, Zhang et al. 14] improved dGCF to FGCF (fast geodesic curvature flow) by reducing the problem dimension. Both dGCF and FGCF require numerical solvers and the computation result is sensitive to the user specified parameters (e.g., convergence tolerance). In sharp contrast, our geodesic loop algorithm is parameterfree and does not require any numerical solver. Furthermore, it can deform an arbitrary curve into exact geodesic loop within finite steps. The experimental results show that our method is much faster than dGCF [11] and FGCF [14].

\section{Our Algorithm}

In this section, we present the algorithm for computing 3 tunnel loops and 3 handle loops for the VS models, which can be paired into $\left(a_{i}, b_{i}\right), i=1,2,3$. The spectrum determined by their lengths can be used for AIS detection purpose. The exact description of tunnel loops and handle loops is available in [2].

Overview. Given a vestibular system modeled by a triangular mesh of genus $g(=3)$, we compute the geodesic tunnel and handle loops, which form the homology basis, and then the geodesic spectra with the following steps (see Figure 10): Step 1: For every mesh vertex $v_{i}$, we compute a system of loops: $\mathcal{L}_{i}=\left\{l_{i}^{1}, l_{i}^{2}, \ldots, l_{i}^{2 g}\right\} ;$ and then we obtain $2 g n$ loops.

Step 2: Sort the loops of $\mathcal{L}$ by length in the ascending order;

Step 3: Find $2 g$ representative loops $l_{1}, l_{2}, \cdots, l_{2 g}$ from $\mathcal{L}$ such that they form a homology basis;

Step 4: Evolve $l_{1}, l_{2}, \cdots, l_{2 g}$ into exact closed geodesic loops $\widehat{l_{1}}, \widehat{l_{2}}, \cdots, \widehat{l_{2 g}}$; Step 5: Match $\widehat{l_{1}}, \widehat{l_{2}}, \cdots, \widehat{l_{2 g}}$ into $g$ pairs and compute the geodesic spectra.

Computing a system of loops restricted on mesh edges. Given a closed, oriented surface $M$ of genus $g$, the system of loops [10] is a set of $2 g$ simple loops sharing a common base point. By cutting along a system of loops, $M$ is topologically equivalent to a disk. Erickson and Whittlesey 3 devised an efficient $O(n \log n)$-time algorithm for an 1-skeleton of a surface, i.e., the graph of edges embedded in the polyhedral surface ( $n$ is the number of mesh vertices). Given a source vertex $s$, we compute a system of loops rooted at $s$ by (see Figure 2): 
1. Using Dijkstra's algorithm on the embedded graph $G$, we obtain a shortest path tree $T$ that encodes parent-child relationships rooted at $s$. Then we set a flag "STOP" for edges on $T$ and a flag "PASS" for other edges.

2. We take each face as a node and define two faces $f_{1}, f_{2}$ to be neighbors if they share a "PASS" edge $e=v_{1} v_{2}$. At the same time, we set the weight between $f_{1}$ and $f_{2}$ to be $\|e\|+d\left(v_{1}\right)+d\left(v_{2}\right)$, where $d\left(v_{i}\right)$ is the shortest distance from $s$ to $v_{i}, i=1,2$. This induces a weighted connected graph $G^{*}$.

3. We compute the maximum spanning tree of $G^{*}$, say $T^{*}$, in $O(n \log n)$ time.

4. There are $2 g$ mesh edges that are in neither $T$ nor $T^{*}$. For each edge $e=\overline{v_{1} v_{2}}$ that belongs to neither $T$ nor $T^{*}$, we compute a loop:

$$
\Pi \overline{\bar{v}_{1} v_{2}}=\Pi\left(s, v_{1}\right) \bigcup \overline{v_{1} v_{2}} \bigcup \Pi\left(v_{2}, s\right),
$$

where $\Pi\left(s, v_{1}\right)$ and $\Pi\left(v_{2}, s\right)$ are the shortest paths, from $s$ to $v_{1}$ and $v_{2}$, respectively, computed by Dijkstra's algorithm.

Identifying handle and tunnel loops. After finding a group of closed curves $\overline{\mathcal{L}}$, serving as generators, from the loop set $\mathcal{L}$, we extract $2 g$ curves that can be matched into $g$ pairs. Then, we need to differentiate handle and tunnel loops.

Checking homotopic curves. Among the $2 g n$ loops, many are not homotopic to each other. To efficiently check if two curves are not homotopic, we have the following observation: Let $\mathcal{C}_{1}$ and $\mathcal{C}_{2}$ be two non-self-intersecting closed curves restricted on mesh edges and they do not intersect. Let $f: V \rightarrow \mathbb{R}$ be a smooth function defined on the VS mesh vertices. Then $d f: E \rightarrow \mathbb{R}$ is a one-form defined on each oriented edge. If the given two curves $\mathcal{C}_{1}$ and $\mathcal{C}_{2}$ are homologous, then $\mathcal{C}_{1}-\mathcal{C}_{2}$ bounds a surface patch, say $\partial D=\mathcal{C}_{1}-\mathcal{C}_{2}$. As a result, $\int_{\mathcal{C}_{1}} d f-\int_{\mathcal{C}_{2}} d f=$ $\int_{\mathcal{C}_{1}-\mathcal{C}_{2}} d f=\int_{\partial D} d f=\int_{D} d d f=0$. The last two equations come from the Stoke's theorem and the fact that $d \circ d=0$. Thus, if $\int_{\mathcal{C}_{1}} d f \neq \int_{\mathcal{C}_{2}} d f$, the two curves are not homologous, which implies that they are not homotopic either.
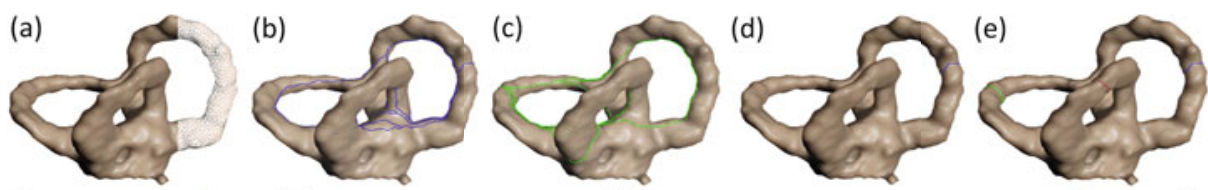

(f)
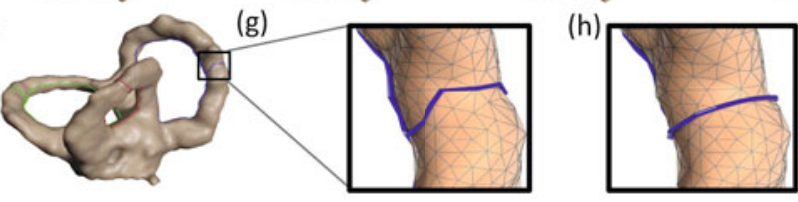

(i)

Fig. 1. Algorithm pipeline. Taking a mesh model of a vestibular system as the input (see (a)), we compute a system of loops for each mesh vertex (see (b) and (c) for two examples). As a result, we obtain $2 g n$ loops $\mathcal{L}$, where $n$ is the number of mesh vertices and $g=3$ the genus of VS. In an incremental manner, we compute the optimal homology basis $\mathcal{L}^{*} \subset \mathcal{L}$ with $2 g$ loops by gradually adding the loops from short to long (d-f). Finally, we evolve each curve in $\mathcal{L}^{*}$ into an exact geodesic loop (i). The close-up views in $(\mathrm{g})$ and $(\mathrm{h})$ reveal the difference between a curved loop and an exact geodesic loop, where the latter has minimal length. 

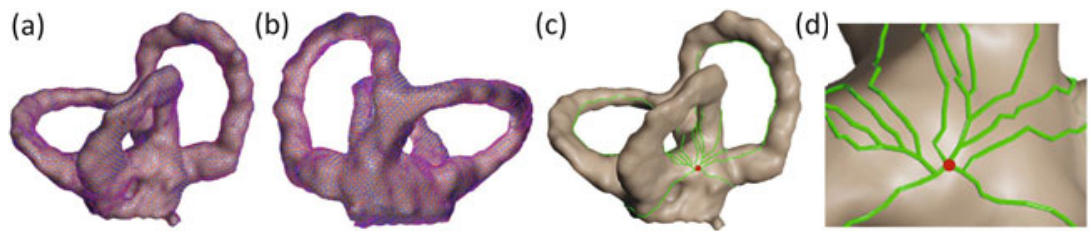

Fig. 2. A system of loop rooted at a given vertex: (a) and (b) show the front and back views of the shortest path tree and the dual graph. (c) shows an example system of loops with $2 g$ curves sharing a common base point. (d) shows the close-up view.
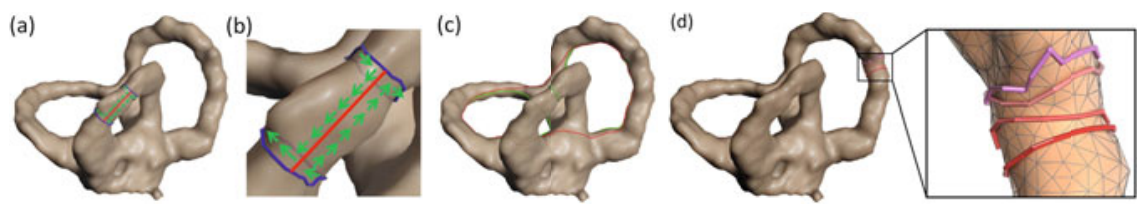

Fig. 3. The two blue loops in (a) are homotopic since one curve can be continuously deformed to the other along the red path (see (b) for the close-up view). The red loop in (c) can be reduced by the three green loops. (d) Our algorithm can deform an arbitrary closed curve (in pink) into an exact geodesic loop (in red) within finite steps. The intermediate results are shown in the close-up view.

The above test can efficiently eliminate a large number of cases where two curves are not homotopic. For curves that pass this test, we use the following approach to test whether they are homotopic. Let $\mathcal{P}\left(\mathcal{C}_{1}, \mathcal{C}_{2}\right)$ be the shortest path (restricted on mesh edges) between $\mathcal{C}_{1}$ and $\mathcal{C}_{2} 1$ Clearly, if the surface patch generated by $\mathcal{C}_{1} \cup \mathcal{C}_{2} \cup \mathcal{P}\left(\mathcal{C}_{1}, \mathcal{C}_{2}\right)$ is a topological disk, then $\mathcal{C}_{1}$ and $\mathcal{C}_{2}$ are homotopic, and thus, we can continuously transform one curve into the other.

Curve reduction. For two homotopic closed curves, we say the longer loop can be reduced by the shorter one; see Figure 3 (a). If the composite loop $l_{a} \bigcup l_{b} \bigcup \mathcal{P}\left(l_{a}, l_{b}\right)$ is homotopic to a loop $l_{c}$ and $\left\|l_{c}\right\|<\left\|l_{a}\right\|$, we say that $l_{a}$ can be reduced into $l_{c}$ by $l_{b}$, where $\mathcal{P}\left(l_{a}, l_{b}\right)$ is the shortest path connecting $l_{a}$ and $l_{b}$. Generally, we can try to reduce a new loop $l_{i}$ by existing loops $l_{j}(1 \leq j<i)$ in order. The goal of curve reduction is to transform the new loop such that it is not homotopic to any one of the existing loops and as short as possible. Figure 3 (c) shows an example where the red loop can be completely reduced by the three green loops (in any reduction order).

Generators and homology basis. We first sort the loops in $\mathcal{L}$ by length, extract the shortest one, say $l_{1}$, and append it into an empty set $\overline{\mathcal{L}}$. For the second shortest loop $l_{2}$, we try to reduce it by the existing loops in $\overline{\mathcal{L}}\left(l_{1}\right.$ in this case). If $l_{2}$ cannot be reduced by any loop in $\overline{\mathcal{L}}$, we append it into $\overline{\mathcal{L}}$. In an incremental style, we can extract a group of closed curves from $\mathcal{L}$ that serve as generators that has $2 g$ closed curves. We observe that for geometrically simple models, the resulting

${ }^{1}$ The "multi-source-all-destination" Dijkstra's algorithm is applied to find the shortest path between $\mathcal{C}_{1}$ and $\mathcal{C}_{2}$ (see Figure 3 (a)). 

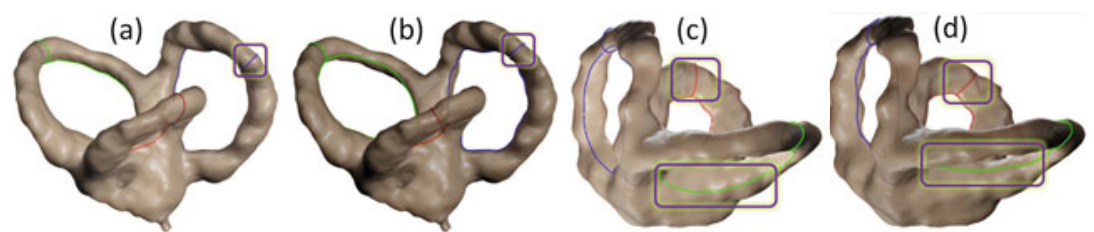

Fig. 4. Comparison to geodesic spectra under hyperbolic metric 13. (a) and (c) show result of 13 . (b) and (d) show our result. The boxes highlight the difference.

generators computed in such a greedy scheme are exactly an optimal homology basis with $g$ pairs that satisfy (1) each pair of loops has a common point; (2) loops in different pairs have no common points; and (3) the total length is minimum in the 1-skeleton of the input mesh. For complicated geometric models, universal covering space 13 should be considered.

Handle and tunnel loops. A closed oriented surface $M$ embedded in $\mathbb{R}^{3}$ partitions $\mathbb{R}^{3}$ into interior sub-space $I$ and exterior sub-space $O$. Tunnel and handle loops are special homology basis that span a surface in the complement space of the input surface. Specifically, a handle loop (tunnel loop) is null-homologous in $I$ $(O)$ but not in $O(I)$. So we can differentiate handles and tunnels by shrinking the loops a little bit and check if they are inside the mesh.

Deforming loops by shortening the length. Given a closed curve $\mathcal{C}$ restricted on mesh edges, we will deform it into an exact geodesic loop (generally not restricted on mesh edges) that is locally shortest on the polyhedral surface. The two key operations are 1) computing a shortest loop restricted on a closed face sequence and 2) updating the edge sequence. Note that the deformation proceeds on the surface (including the face interior), rather than only on mesh edges. The detailed algorithm is as follows:

1. Find the closed edge sequence $\Gamma$ that contains $\mathcal{C}$ and build a loop set $\overline{\mathcal{L}}=\emptyset$;

2. Cut $\Gamma$ along one edge $e=v_{1} v_{2} \in \Gamma$ into an open face sequence $\Gamma^{\prime}$ and unfold it onto a $2 \mathrm{D}$ plane;

3. Since $\Gamma^{\prime}$ encloses a simple polygon $\mathcal{G}$, we can find the shortest path, inside $\mathcal{G}$, between $v_{1}$ and its image point $v_{1}^{\prime}$, and then add the path into $\overline{\mathcal{L}}$. Similarly, we add the shortest from $v_{2}$ into $\overline{\mathcal{L}}$, as well as the image point of $v_{2}$.

4. By traversing all edges in $\Gamma$, we can add more loops into $\overline{\mathcal{L}}$;

5. Replace the loop $\mathcal{C}$ with the shortest loop $\mathcal{C}^{\prime}$ in $\overline{\mathcal{L}}$. (Note: $\mathcal{C}^{\prime}$ is shorter than $\mathcal{C}$.)

6. If $\mathcal{C}^{\prime}$ passes through some vertex $v$ in $\Gamma$, then update $\Gamma$ from one side of $v$ to the other side to test whether the new edge sequence can give a shorter loop, and go to Step 2; otherwise, output the resultant loop.

It is clear that the resultant loop is a closed polyline through an alternative sequence of edges and vertices, and it can be proved that the resultant loop is locally shortest everywhere and shorter than the initial loop. The algorithm is highly efficient and outperforms previous algorithms; see [12] for detail. 


\section{Experimental Results}

Image acquisition and Segmentation of VS: Experiments were done on 11 girls with right-thoracic AIS (mean age 15 years old with variance 1.7 years old); (mean Cobb's angle 27.27 degrees with variance 15.62 degrees) and 11 agematched healthy girls. VS surface meshes were extracted from the T2-weighted MRI scanning of the inner ears using the 1.5T MR Scanner (Sonata, Siemens, Erlangen, Germany) with a quadrature head coil.

Computation of Exact Geodesic Loops: Our proposed algorithm can deform any closed loop to an exact geodesic loop within finite number of steps; we experimented it with a large number of $3 \mathrm{D}$ models and the statistical results showed that the complexity is roughly linear to the number of vertices inbetween the initial and resultant loops. For the given VS meshes with roughly $13 \mathrm{~K}$ triangles, our entire algorithm (including computation of the systems of loops, identification of $2 g$ homology basis, and deforming them into geodesic loops) takes less than 1 second on a workstation with an Xeon $2.66 \mathrm{GHz} \mathrm{CPU}$ and 4GB RAM. The hyperbolic Ricci flow approach [13, in sharp contrast, is a highly non-linear PDE with high computational cost. Our results show that our method is at least one magnitude order of faster than 13] (see also Figure 44).

Exact Geodesic Loops for the Morphometry of VS: We have computed the exact geodesic loops on the VS of the healthy and AIS groups. Figure[5]shows some examples, where the upper row are some normal VS models while the lower row are some AIS VS models. The statistics of the geodesic loops $\left(a_{i}, b_{i}\right)$ 's on the VS from each groups are also as shown in Table1. The statistical difference in the geodesic loops between groups are also evaluated using $t$ tests. Basically, a larger $a_{i}$ means the canal is longer, whereas a larger $b_{i}$ means the canal is thicker. Notice that the exact geodesic loops are computed based on the real background (Euclidean) metric. It is different from 13 in which the lengths of features are computed based on the hyperbolic metric. Hence, our algorithm describes the actual geometry of the VS. From the statistics, AIS tends to have larger $a_{i}$ 's (hence longer canals) and smaller $b_{i}$ 's (hence thinner canals) than the normal groups. In particular, AIS tends to have larger $a_{1}$ and $a_{3}$ with very high statistical significance (both with $\mathrm{P}$-value $<0.02$ ). This implies the lateral and superior canals are generally longer for AIS subjects. The ratio $a_{1} / b_{1}$ and $a_{3} / b_{3}$ also tend to be larger in the AIS group with high statistical significance $(\mathrm{P}=0.0083$ and $\mathrm{P}=0.0321$ ). It means the conformal modules of the lateral and superior canals are significantly different between groups. This implies once again that the shape in the lateral and superior canals tends to be significantly different between the two groups. Compared to [13, our new algorithm can detect more geometric differences between groups with high statistical significance. Also, the P-value for the detected shape differences is much smaller than that in [13. It means our extracted feature curves can conclude shape differences with much higher statistical significance. We also ran a multiple-comparison test. The results in the test agree with our findings that the shape in the lateral and superior canals tends to be different more significantly between the AIS and healthy groups. 


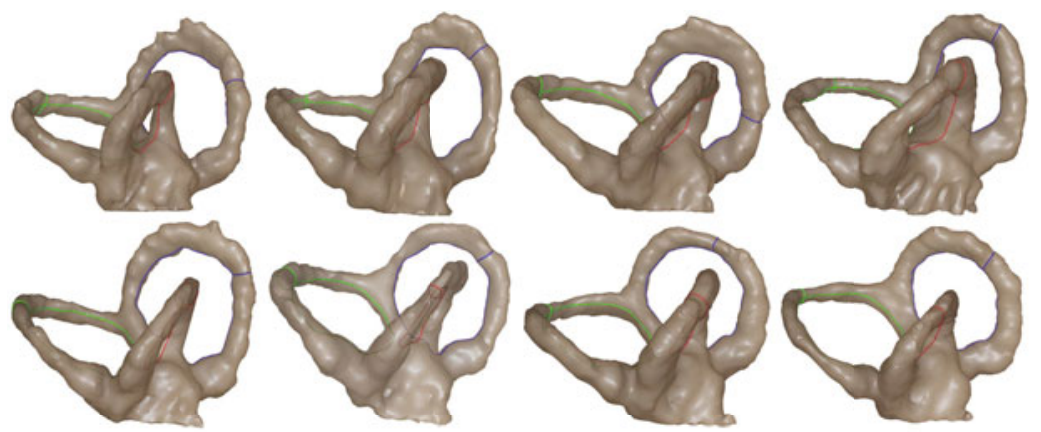

Fig. 5. More results. Row 1: normal subjects; Row 2: AIS subjects

Table 1. Statistics on the exact geodesic loops between the normal and AIS groups

\begin{tabular}{c|ccc}
\hline Combination & Mean(Normal) & Mean(AIS) & P-value \\
\hline$a_{1}$ & $8.7835 \pm 1.2119$ & $9.8544 \pm 0.6469$ & $\mathbf{0 . 0 1 7 7}$ \\
$a_{2}$ & $16.4447 \pm 1.3652$ & $17.1932 \pm 0.9952$ & 0.1573 \\
$a_{3}$ & $14.8008 \pm 1.2991$ & $16.2392 \pm 1.1075$ & $\mathbf{0 . 0 1 1 2}$ \\
$b_{1}$ & $3.8099 \pm 0.3643$ & $3.4912 \pm 0.5146$ & 0.1092 \\
$b_{2}$ & $3.0688 \pm 0.4108$ & $2.8679 \pm 0.3759$ & 0.2455 \\
$b_{3}$ & $3.4521 \pm 0.4980$ & $3.2118 \pm 0.3699$ & 0.2135 \\
$a_{1} / b_{1}$ & $2.3185 \pm 0.3543$ & $2.8882 \pm 0.5390$ & $\mathbf{0 . 0 0 8 3}$ \\
$a_{2} / b_{2}$ & $5.4207 \pm 0.6631$ & $6.1018 \pm 0.9580$ & 0.0668 \\
$a_{3} / b_{3}$ & $4.3743 \pm 0.7917$ & $5.1234 \pm 0.7322$ & $\mathbf{0 . 0 3 2 1}$ \\
$a_{1}+a_{2}+a_{3}$ & $40.0290 \pm 2.7322$ & $43.2868 \pm 2.2105$ & $\mathbf{0 . 0 0 6 0}$ \\
$b_{1}+b_{2}+b_{3}$ & $10.3309 \pm 0.8356$ & $9.5710 \pm 0.8810$ & 0.0510 \\
$a_{1}+b_{1}+a_{2}+b_{2}+a_{3}+b_{3}$ & $50.3599 \pm 3.2353$ & $52.8578 \pm 2.6241$ & 0.0606 \\
\hline
\end{tabular}

\section{Conclusion}

This work proposes an effective method that extracts exact geodesic loops on high-genus surfaces to support analysis of feature landmarks on the VS. The extracted loops are computed using the Euclidean metric, and hence can be used as geometric features to detect shape differences between genus-3 VS. Our proposed method is fully automatic and highly efficient. We applied it to the VS of normal and right-thoracic AIS subjects; experimental results reveal geometric differences in the two groups with high statistical significance.

Acknowledgements. This project was partially supported by NRF2008IDMIDM004-006, AcRF 69/07 and HK RGC grant (ID: 411910).

\section{References}

1. Byl, N., Gray, J.: Complex balance reactions in different sensory conditions: adolescents with and without idiopathic scoliosis. Journal of Orthopaedic Research 11(2), 215-227 (1993) 
2. Dey, T.K., Li, K., Sun, J.: Computing handle and tunnel loops with knot linking. In: Computer Aided Design, vol. 41, pp. 730-738 (2009)

3. Erickson, J., Whittlesey, K.: Greedy optimal homotopy and homology generators. In: Proc. of ACM-SIAM Symposium on Discrete Algorithms, pp. 1038-1046 (2005)

4. Guo, X., Chau, W., Hui-Chan, C., Cheung, C., Tsang, W., Cheng, J.: Balance control in adolescents with idiopathic scoliosis and disturbed somatosensory function. Spine 31, 437-440 (2006)

5. Jin, M., Luo, F., Gu, X.D.: Computing general geometric structures on surfaces using ricci flow. In: Computer-Aided Design, vol. 39(8), pp. 663-675 (2007)

6. Mitchell, J.S.B., Mount, D.M., Papadimitriou, C.H.: The discrete geodesic problem. SIAM J. Comput. 16, 647-668 (1987)

7. Sharir, A., Baltsan, A.: On shortest paths amidst convex polyhedra. In: Proc. of Symposium on Computational Geometry, SCG 1986, pp. 193-206 (1986)

8. Shi, L., Wang, D., Chu, W., Heng, P., Burwell, R., Cheng, J.: Statistical morphometry of the vestibular system in adolescent idiopathic scoliosis. In: Annual World Congress for Brain Mapping and Image Guided Therapy, Harvard Med. Sch. (2009)

9. Simoneau, M., Lamothe, V., Hutin, E., Mercier, P., Teasdale, N., Blouin, J.: Evidence for cognitive vestibular integration impairment in idiopathic scoliosis patients. BMC Neuroscience 10(102) (2009)

10. de Verdière, E.C., Lazarus, F.: Optimal system of loops on an orientable surface. In: Proc. of the Symposium on Foundations of Computer Science, pp. 627-636 (2002)

11. Wu, C., Tai, X.: A level set formulation of geodesic curvature flow on simplicial surfaces. IEEE Tran. on Vis. and Computer Graphics (TVCG) 16, 647-662 (2010)

12. Xin, S.Q., He, Y., Fu, C.W.: Efficiently computing exact geodesic loops within finite steps. IEEE Tran. on Vis. and Computer Graphics, TVCG (accepted, 2011)

13. Zeng, W., Lui, L., Shi, L., Wang, D., Chu, W., Cheng, J., Hua, J., Yau, S., Gu, X.: Shape analysis of vestibular systems in adolescent idiopathic scoliosis using geodesic spectra. In: Jiang, T., Navab, N., Pluim, J.P.W., Viergever, M.A. (eds.) MICCAI 2010. LNCS, vol. 6363, pp. 538-546. Springer, Heidelberg (2010)

14. Zhang, J., Wu, C., Cai, J., Zheng, J., Tai, X.: Mesh snapping: Robust interactive mesh cutting using fast geodesic curvature flow. Computer Graphics Forum (In Proc. of Eurographics 2010) 29(2), 517-526 (2010) 